\title{
AVALIAÇÃO DO APEGO EM ADULTOS: ESTUDO DE REVISÃO INTEGRATIVA DE ESCALAS
}

Gustavo Kastien Tartaro

Makilim Nunes Baptista

Universidade São Francisco
Recebido em: 04/08/2020

$1^{\text {a }}$ revisão em: 06/07/2021

$2^{a}$ revisão em: 05/09/2021

Aceito em: 24/09/2021

\section{RESUMO}

O presente estudo tem como objetivo apresentar uma revisão literatura sobre medidas de apego publicadas no período de 2014 a 2019. Foi realizado levantamento de informações relativas ao número de itens, fatores, forma de resposta, dados de confiabilidade, principais métodos de investigação da estrutura interna. As bases de dados utilizadas foram: BIREME, PubMed, PsycNET, ScienceDirect, Wiley Online Library, SAGE, Springer e Redalyc. Dos 1384 artigos foram analisados 10 que corresponderam aos critérios de inclusão e exclusão. Os fatores os instrumentos apresentaram variações de 1 a 5 fatores e diferentes métodos de análise. Uma lacuna importante encontrada, foi a ausência de um instrumento de apego brasileiro.

Palavras-chave: apego-adulto; revisão de literatura; psicometria; propriedades psicométricas. 


\section{EVALUATION OF ATTACHMENT IN ADULT: SCALES AND PSYCHOMETRIC PROPERTIES}

\section{ABSTRACT}

This study aims to present a literature review on attachment measures published from 2014 to 2019. Information was collected on the number of items, factors, response form, reliability data, main methods of investigation of the internal structure. The databases used were: BIREME, PubMed, PsycNET, ScienceDirect, Wiley Online Library, SAGE, Springer and Redalyc. Of the 1384 articles, 10 that met the inclusion and exclusion criteria were analyzed. The factors of the instruments varied from 1 to 5 factors and different methods of analysis. An important gap found was the absence of a Brazilian attachment instrument.

Keywords: adult attachment; literature review; psychometry; psychometric properties.

\section{EVALUACIÓN DEL APEGO EN ADULTOS: ESCALAS Y PROPIEDADES PSICOMÉTRICAS}

\section{RESUMEN}

Este estudio tiene como objetivo presentar una revisión de la literatura sobre las medidas de apego publicadas de 2014 a 2019. Se recopiló información sobre el número de ítems, factores, formulario de respuesta, datos de confiabilidad, principales métodos de investigación de la estructura interna. Las bases de datos utilizadas fueron: BIREME, PubMed, PsycNET, ScienceDirect, Wiley Online Library, SAGE, Springer y Redalyc. De los 1384 artículos, se analizaron 10 que cumplieron con los criterios de inclusión y exclusión. Los factores de los instrumentos variaron de 1 a 5 factores y diferentes métodos de análisis. Una brecha importante encontrada fue la ausencia de un instrumento de apego brasileño.

Palabras clave: apego adulto; revisión de literatura; psicometría; propiedades psicométricas. 


\section{INTRODUÇÃO}

O conhecimento científico da teoria do apego, desenvolvida por John Bowlby $(1969,1973,1980)$ influenciou as produções sobre o desenvolvimento humano de forma relevante. Através de experiências de proximidade e segurança nos primeiros anos de vida, o autor observou que as crianças desenvolvem crenças acerca do ambiente e suas relações, influenciando sua maturação e desenvolvimento psicossocial, apresentando impactos na vida adulta.

Os primeiros estudos sobre a teoria do apego iniciados por Bowlby, (1969) ganharam novos ares a partir de Ainsworth, Blehar, Waters e Wall, (1978), pois os autores trataram de levar a teoria do apego para o campo experimental observacional, como o experimento chamado de Strange Situation Procedure, que consistia na classificação do estilo de apego em crianças com meses de vida a partir da observação da interação entre elas e suas mães.

Alguns anos depois, surgiram tentativas de sistematização e classificação dos padrões de vinculação na vida adulta, sendo desenvolvido diferentes modelos teóricos com fins de avaliação psicológica. Hazan e Shaver (1987), baseados nos estudos de Ainsworth, operacionalizaram os padrões de vinculação em três tipos, a saber: Secure (seguro), Anxious (ansioso) e Avoidant (evitativo). Já Griffin e Bartholomew (1994), com maior ênfase nas propostas teóricas de Bowlby, realizaram a divisão entre modelo de Self (percepção de si) e modelo Other (percepção sobre os outros), com os estilos de apego Secure (seguro), Preoccupied (preocupado), Fearful (temeroso) e Dismissing (desinvestido).

Cabe ressaltar que a partir destes dois modelos mencionados surgem instrumentos de apego-adulto importantes. Dentre eles, a escala oriunda das contribuições de Hazan e Shaver (1987), chamada de Experiences in Close Relationships (ECR) com ênfase no apego romântico. Posteriormente, com as contribuições de Griffin e Bartholomew (1994), foi criado o Relationships Style Questionnaire (RSQ), com características mais amplas não focalizando apenas relações amorosas. Ambos instrumentos são utilizados internacionalmente até os dias atuais.

Em linhas gerais, os padrões de vinculação segura (Secure) remetem ao cuidado consistente na primeira década de vida e a internalização de aspectos positivos sobre si e o ambiente. Por outro lado, os padrões de vinculação insegura são referentes a um modelo de cuidado pouco sensível ou negligente. Os padrões inseguros geram a necessidade constante de confirmação e busca pela figura de apego (Anxious e Preoccupied) ou resultam na esquiva, medo ou desdém em relação a esta figura (Avoidant, Fearful e Dismissing) (Yip, Ehrhardt, Black, \& Walker, 2018; Cassidy, Jones \& Shaver, 2014).

Inicialmente os estudos empíricos sobre apego focaram-se no desenvolvimento infantil, partindo posteriormente para as demais fases de desenvolvimento (adolescência, idade adulta, velhice) (Ravitz, Maunder, Hunter, Sthankiya \& Lancee, 
2010; Baptista, Tartaro \& Peixoto, 2021). Desta forma, diferente dos métodos observacionais direcionados a crianças de pouca idade, com crianças maiores, adolescentes e adultos os estilos de apego podem ser avaliados técnicas que demandam maior capacidade cognitiva no processo de resposta, como escalas (Baptista et al., 2021).

Apesar de algumas desvantagens como desejabilidade social e aquiescência, o uso de escalas apresentam algumas vantagens, pois são ferramentas de baixo custo financeiro no âmbito da pesquisa e de fácil aprendizagem para aplicação (Greenwald, Nosek \& Sriram, 2006; Blanton 2018). Através do uso das escalas de apego, ao longo das últimas décadas, vários estudos no campo da saúde mental demonstraram associações entre os estilos de apego na vida adulta e outros construtos psicológicos como personalidade, autoestima e depressão na população adulta, os estilos de apego inseguro, de modo geral tendem apresentar piores indicadores de saúde mental (Barnum \& Perrone, 2017; Varghese \& Pistole, 2017; Moshkani \& Afrooz 2018; Özyurt, Öztürk, Onat, Mutlu \& Akay, 2018).

Como se trata de uma área com uma diversidade instrumental com estruturas fatoriais e modelos diferentes, é importante para a prática de avaliação psicológica e de pesquisa obter conhecimento acerca dos instrumentos mais utilizados e de suas características. Tais informações podem permitir e embasar a criação de novos materiais, podendo ser de grande relevância para a prática clínica e de pesquisa (Ravitz et al., 2010).

Para tanto, o objetivo deste trabalho é a realização de uma revisão de literatura integrativa que permita explorar e reconhecer escalas de apego adulto utilizadas no período de 2014 a 2019. É almejado o levantamento de informações de instrumentos de apego, relativas ao número de itens, quantidade de fatores, forma de resposta, dados de confiabilidade, principais métodos de investigação da estrutura interna e informações psicométricas disponibilizadas pelos autores dos estudos incluídos.

\section{MÉTODO}

Para poder corresponder ao objetivo deste estudo, o método escolhido foi o de revisão integrativa de literatura. Como o número de publicações relacionadas ao tema apego é elevado, para a concretização deste trabalho optou-se pela seleção de artigos publicados de 2014 a 2019, pois, tal período permitiria abranger estudos com instrumentos já consagrados pela literatura e também instrumentos criados recentemente, tornando possível assim o mapeamento de algumas das escalas existentes.

Foram utilizados os seguintes critérios de inclusão: estudos psicométricos que focalizaram a investigação de algum tipo de evidência de validade ou de adaptação. Este critério foi escolhido por possibilitar acesso a informações relacionadas à quantidade de itens e fatores, forma de resposta, confiabilidade 
obtida na amostra do estudo e principais métodos de investigação de estrutura interna.

Quanto a língua, foram selecionadas publicações em idioma português, inglês ou espanhol, abrangendo desta forma trabalhos nacionais e internacionais. Como o objetivo foi o de conhecer escalas de apego em adultos, foram removidos estudos que abordaram instrumentos relacionados a apego em crianças, apego maternofetal, paterno-fetal, apego a animal, lugar, aparelho eletrônico ou outros. Teses dissertações e trabalhos de conclusão de curso, livros, resenhas e artigos teóricos também foram excluídos.

Quanto às fontes de informação, a busca dos estudos ocorreu no período de março a junho de 2019 e as bases de dados foram escolhidas por sua abrangência. Com o intuito de aprimorar a busca para a composição da amostra de artigos, os termos foram consultados nos Descritores em Ciências de Saúde (DeCS) e ao Medical Subject Headings (MeSH). Contudo, o termo "attachment" de modo isolado não foi encontrado, assim, optou-se pela aplicação de palavras-chave comumente utilizadas em estudos sobre evidências de validade de instrumentos de apego, pois, desta forma seria possível encontrar correspondência entre os estudos.

A busca por artigos contou, portanto, com os termos: "Attachment", "Scale" e "Psychometric". A combinação das palavras entre título e resumo foram feitas conforme as opções exibidas pelas plataformas de pesquisa, de modo que os termos deveriam estar presentes no título, resumo ou corpo do texto. Foi encontrado 1384 artigos, as referências foram agrupadas com auxílio do software Zotero (Vanhecke, 2008). As informações acerca das bases de dados e o número de artigos podem ser visualizadas na Tabela 1.

\section{Tabela 1.}

Bases de dados, descritores de busca e números de estudos encontrados.

\begin{tabular}{c|c}
$\begin{array}{c}\text { Base de } \\
\text { dados }\end{array}$ & $\begin{array}{c}\text { Número de } \\
\text { referências obtidas }\end{array}$ \\
BIREME & 119 \\
PubMed & 179 \\
PsycNET & 21 \\
ScienceDirect & 42 \\
Wiley Online & 216 \\
Library & \\
SAGE & 130 \\
Springer & 209 \\
Redalyc & 468 \\
Total & 1384 \\
\hline
\end{tabular}


O primeiro procedimento seguido foi a remoção de itens duplicados, reduzindo a amostra para 984 artigos. Após a exclusão das duplicatas, o segundo procedimento realizado foi o de leitura de títulos, resumos e exclusão de artigos que não se enquadravam ao objetivo do estudo, neste processo, restaram 29 artigos. O último procedimento realizado foi o de leitura na íntegra seguindo os critérios de inclusão e exclusão, de modo que restaram ao final, 10 estudos (processo exposto no fluxograma da Figura 1).

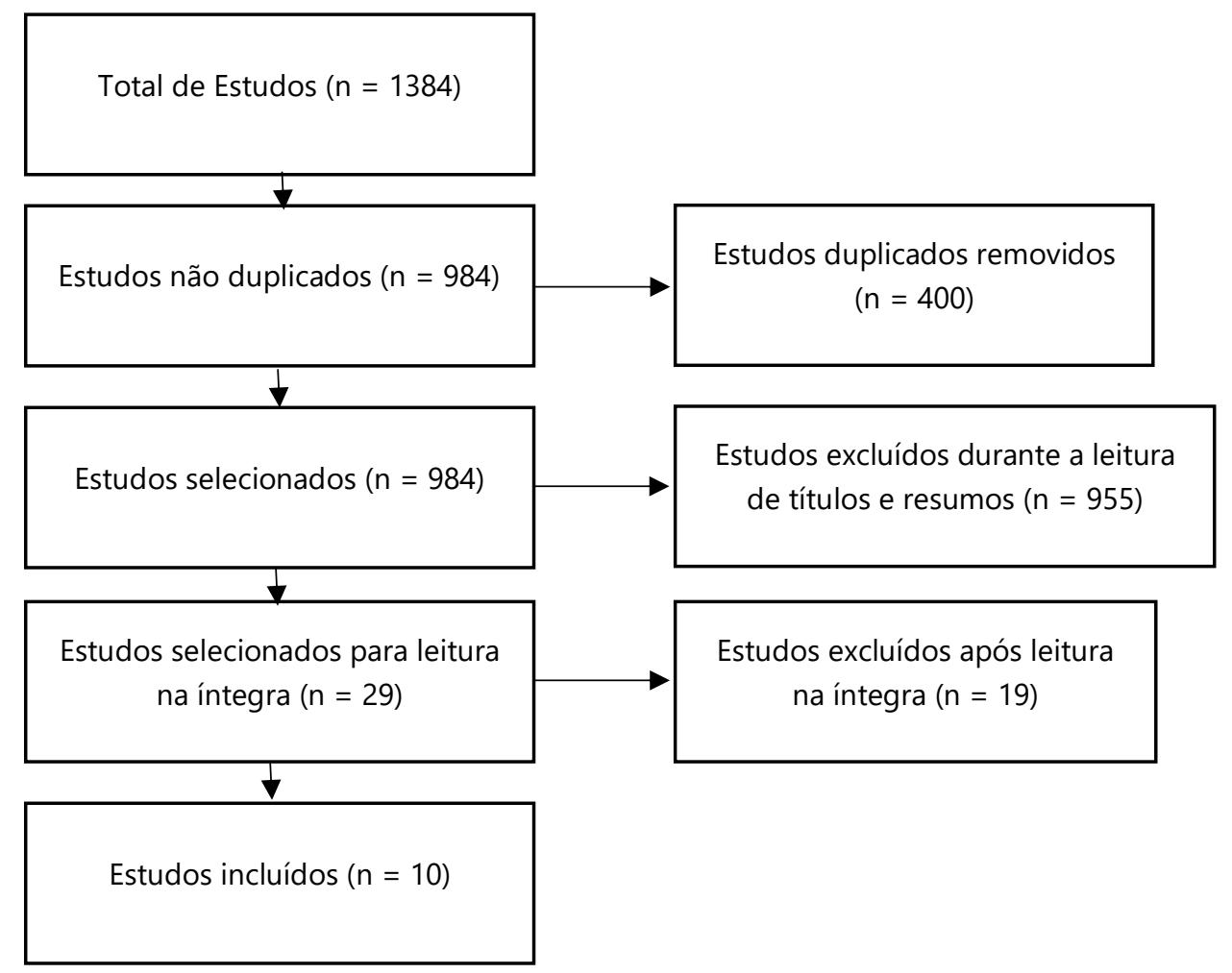

Figura 1 - Fluxograma de Escolha dos Artigos Finais Analisados

Para a realização da extração dos dados e integração das informações obtidas, foi elaborada uma tabela com informações alvo da pesquisa, separada em colunas com nome do instrumento, autoria, número de itens, fatores de apego (quantidade e nome), tipo de resposta, dados de confiabilidade e principais métodos de análise.

Em relação aos estudos encontrados, os fatores dos instrumentos serão descritos com as nomenclaturas apresentadas na íntegra, em inglês, com tradução livre para português (Brasil). O intuito deste feito é que o leitor possa compreender tanto a nomenclatura dos artigos originais, quanto seu equivalente em português. Ademais, a exposição dos resultados seguirá seus respectivos métodos e valores psicométricos explicitados pelos autores que os publicaram, os estudos inclusos na revisão serão indicados com * na lista de referência para auxiliar sua identificação. 


\section{RESULTADOS}

Todos os estudos encontrados apresentam estudos de validação, destes, dois foram realizados com a população brasileira (ECR-RS de Rocha et al., 2017 e URCS de Vilar et al., 2017). A seguir, as escalas serão detalhadas em suas características (Tabela 2).

\section{Tabela 2.}

\section{Instrumentos encontrados durante a revisão}
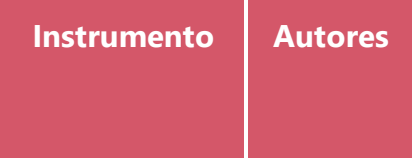

Firoozab

Attachment
Style
Questionnaire

(ASQ-24)

The Adult Scale of Parental AttachmentShort Form

(ASPA-SF)

Relationship Scale Questionnaire

(RSQ)

\section{$\mathrm{n}$,}

Anderse

Pedersen

, Carlsen

Elesen e

Vedsted

(2017)

Abedi,

Aliyari, Zolfagha

Ghanizad

eh (2014)

Michael

(2019)

\begin{tabular}{c|c} 
Iten & Fatores \\
$\mathbf{S}$ & \\
$\left(\mathbf{N}^{\circ}\right)$ &
\end{tabular}

Resposta

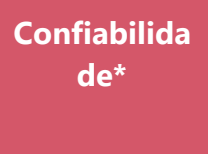

Análises

Seguro,

Temeroso,

$1=$ Discordo

Preocupado e

totalmente a 5

= concordo

Desinvestido. totalmente.

42

$$
\begin{gathered}
\text { Seguro, } \\
\text { Dependente, } \\
\text { Temeroso, } \\
\text { Distante e }
\end{gathered}
$$

Parentificação.

$1=$ Nunca a 5

Constantemen

te.

Encontrados:

Independênci

a,

Preocupação

com

Relacionamen

to,

Proximidade.

Originais:

Seguro,

Preocupado,

Temeroso e

Desinvestido.

\section{Experiences in Busonera \\ Close Martini, \\ Relationships- Zavattini \\ Revised \\ e \\ Santona}

(ECR-R)

Experiences in

Close

Relationships -

Relationship

(2014)

Rocha,

Peixoto,

Nakano,

Motta e

Wiethae

\section{6}

Ansiedade e
Evitação.

1 = Discordo

totalmente a 7

= Concordo

totalmente.

$1=$ Discordo

Ansiedade e totalmente a 7

Evitação. = Concordo

totalmente.
$0,58 / 0,76$

ACP

0,69/0,90 AFC

$0,73 / 0,78 \quad$ AFE

0,79/0,88 AFC

TRI 


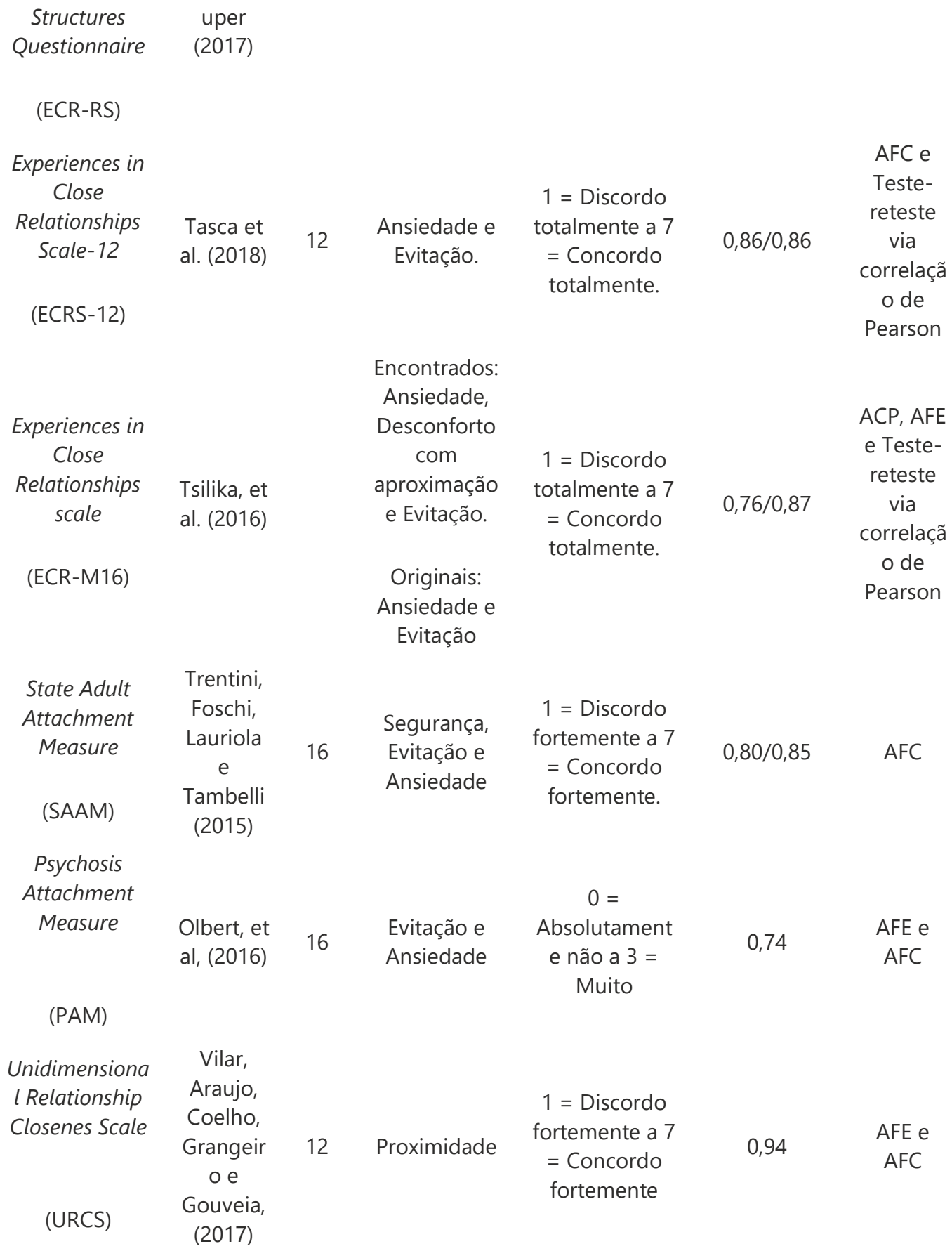

Nota. *Mínimo e máximo considerando o coeficiente $\alpha$ encontrado para os fatores. AFE = Análise Fatorial Exploratória; AFC = Análise Fatorial Confirmatória; TRI = Teoria de Resposta ao Item; ACP = Análise de Componentes Principais.

\section{ASQ-24}

A escala ASQ-24, é uma versão curta da ASQ de Feeney, Noller e Hanharan (1994), esta versão apresenta 24 itens em escala Likert com pontos de $1=$ Discordo totalmente a 5 = Concordo totalmente, possui os fatores: Secure, Fearful, Preoccupied e Dismissing. No estudo de adaptação de Firoozabadi et al. (2014) para a população iraniana, foi utilizada ACP. O teste de esfericidade de Bartlett 
demonstrou a adequação para os dados (KMO $=0,817 ; p=<0,001)$. Os autores analisaram a estrutura interna da escala via ACP sendo utilizado como método de rotação oblimin. Foi encontrada a adequação de quatro fatores extraídos explicando $44,94 \%$ da variância total. Na amostra foi encontrado um coeficiente $\alpha$ de 0,63 para o fator Secure, 0,76 para Fearful, 0,72 para Preoccupied e 0,58 para Dismissing.

\section{ASPA-SF}

A escala ASPA-SF é uma versão reduzida da ASPA (Snow, Sullivan, Martin, \& Helm, 2005) que possui, em sua versão completa, 84 itens dispostos em escala Likert de 5 pontos em que 1 = Nunca e $5=$ Constantemente. Propõe avaliar apego com base na percepção das relações parentais. Há no instrumento duas subscalas, chamadas de Mother e Father, dispostas com os fatores: Safe, Dependent, Fearful, Distant e Parentified. No estudo incluído, feito por Michael e Snow (2019) foram selecionados 42 itens do instrumento original para a composição de uma versão breve do instrumento, a estrutura interna da escala criada foi acessada através de AFE com o método Principal Axis Factoring, primeiro com a subescala Mother e depois com a subescala Father.

Foram extraídos 5 fatores em ambas subescalas, explicando 43,6\% e 48, 08\% da variância (Mother/Father respectivamente). Após este procedimento os autores realizaram uma Análise Fatorial Confirmatória (AFC), apresentando índices de ajuste para a escala Mother: $\chi 2=274,46(\mathrm{gl}=160) ; \mathrm{CMIN} / \mathrm{DF}=1,715 ; \mathrm{CFI}=0,93$; $\mathrm{TLI}=0,91 ; \mathrm{RMR}=0,07 ; \mathrm{RMSEA}=0,05 . \mathrm{E}$ para a escala Father: $\chi 2=395,72(\mathrm{gl}=160) ;$ $\mathrm{CMIN} / \mathrm{DF}=2,47 ; \mathrm{CFI}=0,90 ; \mathrm{TLI}=0,90 ; \mathrm{RMR}=0,10 ; \mathrm{RMSEA}=0,07)$. Na amostra o coeficiente $\alpha$ variou de 0,73 a 0,88 para a subescala Mother e 0,69 a 0,90 para a subescala Father (Michael \& Snow, 2019).

\section{RSQ}

A RSQ avalia estilos de apego, com 30 itens em escala tipo Likert de 5 pontos, sendo 1 = Nada parecido comigo e 5 = Muito parecido comigo. Sua estrutura fatorial original é composta por 4 fatores, Secure, Preoccupied Dismissing e Fearful (Griffin \& Bartholomew, 1994). O estudo de Andersen et al. (2017), incluído na revisão, consistiu na validação e adaptação transcultural ao contexto dinamarquês. Os autores utilizaram duas amostras: uma de pacientes com câncer e outra com profissionais da saúde, sendo que a estrutura de quatro fatores da versão original não pôde ser mantida devido ao não ajuste dos índices ao modelo.

Os autores dividiram as amostras aleatoriamente mantendo em tamanhos iguais para a realização de AFE e AFC. Por meio da AFE foram encontrados 3 fatores (Independence, Relationship Worry e Closeness). Apenas 17 dos 30 itens apresentaram adequação ao modelo (Amostra de clínicos gerais $\chi 2=180,751$ (df $=52) ; \mathrm{CFI}=0,945 ; \mathrm{TLI}=0,904 ; \mathrm{RMSEA}=0,093[\mathrm{Cl}=0,079,0,108]$; $\mathrm{SRMR}=0,045$; Amostra de pacientes: $\chi 2=199,549(\mathrm{gl}=52) ; \mathrm{CFI}=0,922 ; \mathrm{TLI}=0,863 ; \mathrm{RMSEA}=$ $0,100[\mathrm{Cl}=0,085 ; 0,114]$; SRMR $=0.049$ ). 
O modelo alternativo foi também testado via AFC (Amostra de clínicos gerais $\chi 2=$ $239,065$ ( $\mathrm{gl}=74) ; \mathrm{CFI}=0,922 ; \mathrm{TLI}=0,904 ; \mathrm{RMSEA}=0,088[\mathrm{Cl0}, 076 ; 0,100]$. Amostra de pacientes $278 \times 2=181,703(\mathrm{gl}=74) ; \mathrm{CFI}=0,927 ; \mathrm{TLI}=0,911$ RMSEA 0,072 $[\mathrm{Cl}$ $0,059 ; 0,086])$. A confiabilidade via coeficiente $\alpha$, para a amostra de pacientes foi de 0,73 a 0,74 e de 0,77 a 0,78 para amostra de clínicos gerais.

\section{ECR-R}

A escala ECR-R de Busonera et al. (2014) é uma versão adaptada para população italiana da escala ECR-R de Fraley, Waller e Brennan (2000). É composta por 36 itens em escala tipo Likert de 7 pontos em que 1 = Discordo totalmente e 7 = Concordo totalmente. Os autores realizaram a divisão da amostra em duas partes iguais. Para a primeira parcela foi realizada a extração de cinco fatores iniciais via Principal Axis Factoring, com método de rotação oblimin obtendo cinco fatores (eingenvalues: 7,$1 ; 3,1 ; 1,8 ; 1,7$ e 1,4 respectivamente), mas forçaram a extração de dois fatores que explicaram 28,41\% da variância: Anxiety e Avoidance, correspondentes ao modelo teórico. Para a AFC, segundo os autores, não houve um ajuste totalmente satisfatório, possivelmente por conta do processo de tradução $(\chi 2=364,91$; $(\mathrm{gl}=$ 53); $\mathrm{SRMR}=0,07 ; \mathrm{CFI}=0,95 ; \mathrm{NNFI}=0,94 ; \mathrm{RMSEA}=0,093, \mathrm{IC} 95 \%=0,082 ; 0,103$; $\mathrm{AIC}=414,91 ; p=<0,001)$. Na amostra o coeficiente $\alpha$ encontrado foi de 0,88 para o fator Anxiety e 0,79 para Avoidance.

\section{ECR-RS}

A ECR-RS de Rocha et al. (2017), é composta por quatro escalas de nove itens cada (totalizando 36 itens), dispostas em escala tipo Likert de 7 pontos em que $1=$ Discordo totalmente e $7=$ Concordo totalmente. Foi adaptada ao contexto brasileiro tomando como base a escala ECR-RS (Fraley, Heffernan, Vicary, \& Brumbaugh, 2011), sendo que o instrumento tem como objetivo avaliar o vínculo em relacionamentos com pessoas próximas, em questão: pai, mãe, companheiro íntimo e amigo, ou seja, uma escala destinada para cada figura de vinculação. Para a estimação do número de fatores foi realizada análise paralela utilizando o método Minimum Rank Factor Analysis, indicando 2 fatores (Anxiety e Avoidance), para cada uma das escalas (Mother, Father, Partner, Friends), com variância acumulada de 57,55\%, 59,39\%, 54,82\%, 59,18\%, respectivamente.

A estrutura interna foi avaliada via AFE utilizando o método de estimação Unweighted Least Squares (ULS) e rotação obliqua promax (Escala Mother Bartlett $\chi^{2}=987,9 ; \mathrm{KMO}=0,714 ; p=<0.01 ; /$ Father Bartlett $\chi^{2}=1006,8 ; \mathrm{KMO}=0,727 ; p$ $=<0,01 /$ Partner Bartlett $\chi^{2}=760.6 ; \mathrm{KMO}=0,732 p=<0.01 /$ Friends $\mathrm{KMO}=0,727$ Bartlett $\left.\chi^{2}=907,5 p=<0.01\right)$. Neste mesmo estudo foi realizado ainda a análise de Teoria de Resposta ao Item (TRI), utilizando o modelo de Rasch-Masters Partial Credit Model, fazendo o uso do método estimador Maximum Likelihood. Resultados encontrados pelos autores:

Subescala Mother fator Avoidant Infit/Outfit: $\mathrm{M}=0,99 / 0,91$; $\mathrm{DP}=0,26 / 0,29 ; \mathrm{Max}=$ 0,41/1,47; Min = 0,68/0,66. Fator Anxious Infit/Outfit $\mathrm{M}=1,01 / 0,92 ; \mathrm{DP}=0,27 / 0,22$; 
Min = 1,25/1,10; Min = 0,65/0,61. Subescala Father fator Avoidant: Infit/Outfit: $M=$ 1,01 /1,01; DP = 0,2/0,24; $\operatorname{Max}=1,24 / 1,43 ; \operatorname{Min}=0,67 / 0,64$. Fator Anxious: Infit/Outfit $\mathrm{M}=1,01 / 0,92 ; \mathrm{DP}=0,21 / 0,16 ; \mathrm{Max}=1,23 / 1,05 ; \mathrm{Min}=0,73 / 0,70$.

Subescala Partner Infit/Outfit fator Avoidant: $\mathrm{M}=1,01 / 0,98 ; \mathrm{DP}=0,26 / 0,28 ; \mathrm{Max}=$ 1,37/1,42; Min = 0,7/0,62. Fator Anxious: $\mathrm{M}=1,01 / 0,95 ; \mathrm{DP}=0,15 / 0,13 ; \mathrm{Max}=$ 1,5/1,08; Min = 0,8/0,76. Subescala Friend Infit/Outfit fator Avoidant: $M=1,01 / 1,01$; $\mathrm{DP}=0,3 / 0,37 ; \mathrm{Max}=1,43 / 1,48$. Fator Anxious $\mathrm{M}=1,01 / 0,99 ; \mathrm{DP}=0,19 / 0,17 ; \mathrm{Max}$ $=1,27 / 1,21 ;$ Min $=0,83 / 0,81$. O coeficiente $\alpha$ encontrados na amostra para as subescalas foram de Avoidant/Anxious = 0.87/0.91 (Mother); 0,86/0,91 (Father); 0,78/0,89 (Partner); 0,85/0,88 (Friend).

\section{ECRS-12}

A ECRS-12 estudada por Tasca et al. (2018) é uma escala de 12 itens de autorrelato, voltada para o apego a parceiros românticos. Possui dois fatores de apego, Avoidance e Anxiety em uma escala tipo Likert de 7 pontos em que 1 = Discordo totalmente e 7 = Concordo totalmente. Os estudos de estrutura interna foram realizados via AFC $\left(\chi^{2}(53)=8,3\right.$; RMSEA = 0,076 (90\% Cl: 0,069-0,083); CFI = 0,95; $\mathrm{TLI}=0,94$; Gamma Hat $=0,95 ;$ RMSR $=0,63$ ) sendo que os parâmetros foram estimados utilizando o método Maximum Likelihood. A confiabilidade da escala via $\alpha$ de Cronbach para ambos os fatores foi de 0,86 . Os autores ainda tiveram o cuidado de utilizar com 128 participantes o método de test-retest no decorrer de 160 dias, e puderam observar correlações positivas entre os primeiros e últimos escores (Anxiety: $r=0,71, p=<0,001$; Avoidance $r=0,75, p=<0,001$ ).

No teste de validade convergente a ECR-12, demonstrou correlação positiva com os fatores da ASQ (Feeney et al., 1994), Anxiety e Need for Approval ( $r=0,31 ; p=$ $<0,05)$ e Anxiety e Preoccupation with Relationships $(r=0,49 ; p=<0,01)$, ambos relacionadas à ansiedade de apego. Apresentou ainda correlação positiva entre Avoidance e Discomfort with Closeness ( $r=0,42 ; p=\langle 0,01)$; Avoidance e Relationships as Secondary Scale $(r=0,25 ; p=<0,5)$, ambos, conceitualmente relacionados à evitação. $O$ fator Avoidance correlacionou ainda negativamente ao fator Confidence $(r=-0,28 ; p=<0,05)$.

Os autores ainda separaram cinco grupos diagnósticos de transtornos alimentares para a realização de uma AFC multigrupo, encontrando efeito estatisticamente significativo do grupo diagnóstico na combinação linear das variáveis dependentes (Grupos: tipo restritivo à anorexia nervosa; anorexia nervosa-bingin/purging; bulimia nervosa; compulsão alimentar periódica; transtorno alimentar não especificado, obtendo como resultados: Wilks $\lambda=0,967, F(8,2512)=5,383 ; p=<$ 0,$\left.001 ; h^{2}=0,017\right)$.

\section{ECR-M16}

A escala ECR-M16 foi adaptada para o contexto grego por Tsilika et al., (2016), com amostra de pacientes em cuidados paliativos. O instrumento, como outras versões 
da ECR objetiva avaliar as relações ao apego romântico com 16 itens dispostos em escala tipo Likert em que 1 = Discordo totalmente e 7 = Concordo totalmente. Os autores ao investigarem as propriedades psicométricas para a escala, apresentaram em seus resultados a AFE e ACP como uma análise única, todavia, são procedimentos diferentes e que produzem índices diferentes (ver discussão).

De todo modo, Tsilika et al. (2016) relatam a retenção de 3 fatores que explicaram 58,76\% da variância total: Anxiety, Discomfort with closeness e Avoidance, mencionam ainda a utilização do método de rotação promax, e não apresentam índices de ajuste típicos de uma AFE como esfericidade de Barlett, $\chi 2, \mathrm{KMO}, \mathrm{CFI}$, TLI, RMSEA. Com uma parte da amostra selecionada aleatoriamente, foi realizado test-retest com 7 dias após a primeira avaliação, e os resultados sugeriram correlação significativa ( $p=<0,000$ ), entretanto não foi apresentada a magnitude e direção desta correlação. A consistência interna foi acessada via coeficiente $\alpha$ : 0,76 para o fator Anxiety, 0,87 para Discomfort with closeness e 0,76 para Avoidance.

\section{SAAM}

A escala SAAM de Trentini et al. (2015) é uma escala de apego-adulto, sendo que a publicação dos autores retrata a versão traduzida e adaptada para a população italiana da escala SAAM de Gillath, Hart, Noftle e Stockdale, (2009). Possui 21 itens em escala tipo Likert de 7 pontos em que 1 = Discordo fortemente e $7=$ Concordo fortemente. $O$ instrumento possui 3 fatores: Security, Avoidance e Anxiety. Os dados da escala foram acessados através de AFC $(\chi 2=322,51$ ( $\mathrm{gl}=186)$; NNFI = 0,91; CFI $=0,92 ; \operatorname{RMSEA}=0,056 ; 90 \%$ C.I. $[0,045-0,065] p=<0,001)$. A escala apresentou coeficiente $\alpha$ de 0,85 para o fator Security, 0,84 para Anxiety e 0,80 para Avoidance.

\section{PAM}

Com o intuito de avaliar o apego na psicose, a escala PAM possui 16 itens em escala tipo Likert de 4 pontos em que $0=$ Absolutamente não e $3=$ Muito. Possui originalmente 2 fatores, Avoidance e Anxiety (Berry, Wearden, Barrowclough, \& Liversidge, 2006). Olbert et al. (2016) conduziram inicialmente uma análise de cluster hierárquico em que o algoritmo produziu um cluster primário (valor $=3,3$ ) compreendendo 12 de 16 itens e um cluster secundário (valor = 1,6). Buscando esmiuçar as possibilidades da escala, os autores conduziram uma AFE com rotação oblimin indicando a retenção de 5 fatores, mas destes apenas dois fatores se assemelharam ao modelo teórico. A AFC para um modelo de dois fatores demonstrou índices de ajustes não adequados $(\chi 2=274 ;(g l=103), p=0,01)$, $\mathrm{CFI}=0,66$, RMSEA=0,11; $90 \% \mathrm{Cl}[0,094 ; 0,13])$.

Dada a configuração dos dados da escala, os autores reduziram-na para 6 itens (total), apresentando desta vez uma solução unidimensional apresentando boa correlação com a escala total $(r=0,87 ; p=0,01)$ e coeficiente $\alpha$ de 0,74. Conduziram ainda um procedimento de validade convergente, todavia, não apresentou correlações significativas com outros construtos associados. Os autores 
argumentam que a pouca quantidade de itens final ofereceu baixa contribuição psicométrica para a mensuração do apego.

\section{URCS}

A escala URCS de Vilar et al. (2017) objetiva avaliar o estilo de apego seguro no conforto com proximidade, esta é uma adaptação da escala URCS de Dibble et al. (2012), ao contexto brasileiro. É composta por 12 itens dispostos em escala tipo Likert de 7 pontos em que 1 = Discordo fortemente e 7 = Concordo fortemente, apresentando estrutura unidimensional, em um fator é chamado Closeness. Para avaliar sua estrutura interna unifatorial, uma AFE foi realizada apresentando índices de ajuste $(\chi 2=1639,5(\mathrm{gl}=66) ; \mathrm{KMO}=0,92 ; p<0,001])$, assim como a AFC com estimador Robust Maximum Likelihood ( $\chi 2=120,31$ ( $\mathrm{gl}=54$ ), $p<0,001 ; \mathrm{CFI}=0,937$ $\mathrm{TLI}=0,923 ; \mathrm{SRMR}=0,041 ; \mathrm{RMSEA}=0,072$ [IC90\% =0,055 |0,090]). O coeficiente $\alpha$ encontrado pelos autores na amostra foi de 0,94 .

\section{DISCUSSÃO}

O objetivo deste trabalho foi o de possibilitar a exploração e reconhecimento das escalas de apego utilizadas no período de 2014 a 2019. Foi pretendido realizar o levantamento de informações de instrumentos de apego, relativas ao número de itens, quantidade de fatores, forma de resposta, dados de confiabilidade, principais métodos de investigação da estrutura interna e informações psicométricas disponibilizadas pelos autores dos estudos incluídos. Com base nisto é possível afirmar que os principais objetivos do estudo foram alcançados, ao integrar e disponibilizar informações sobre os instrumentos durante a síntese de resultados.

Um dos aspectos interessantes nas publicações apresentadas é a de que a maioria dos estudos, utilizaram de AFEs e AFCs como método de avaliação da estrutura interna e coeficiente alfa para mensurar a consistência interna dos itens. Em termos de informação, assim como o coeficiente alfa, as AFEs e AFCs possuem alguns índices de ajuste relativo ao modelo teórico e ao modelo encontrado, que tratam de indicar sua plausibilidade ou não. Em ambas as análises, os softwares fornecem alguns valores, como KMO (Kaiser Meyer Olkin) sendo considerado adequado índices acima de 0,70 (Muthén \& Muthén, 2012).

Há também outros índices como os de ajuste CFI (Comparative Fit Index) e TLI (Tucker Lewis Index) em que o valor adequado é acima de 0,90 sendo 0,95 considerado ótimo (Hu \& Bentler, 1999). Para os índices RMSEA (Root Mean Square Error of Aproximation) é esperado valores próximos ou abaixo de 0,05 e SRMR (Standardized Root Mean Square Residual) que reporta de modo padronizado a média, espera-se valores abaixo de 0,10 (Hox \& Bechger, 1998). Já, em relação ao coeficiente de alfa, comumente utilizado para avaliar a consistência interna, o Conselho Federal de Psicologia (CFP, 2018) define como confiáveis instrumentos com valores iguais ou superiores a 0,70. 
Entretanto, é importante salientar que a estimativa do coeficiente alfa pode ser afetada por aspectos relativos ao tamanho amostral e características do público (Souza, Alexandre \& Guirardello, 2017). Neste sentido, a maior porção dos estudos encontrados apresentaram índices de adequados, com destaque negativo à escala PAM (Olbert et al., 2016), o instrumento que visa avaliar apego na psicose apresentou índices expressivamente fora do esperado, é possível que isso tenha ocorrido pela redução brusca de itens e pela condição clínica da amostra. Em sentido oposto, a escala de um fator, URCS (Vilar et al., 2017) destaca-se com melhores índices.

A ECR-R (Busonera et al., 2014) também apresentou complicações para os índices de ajuste, os autores abordam a possibilidade de isto ocorrer devido ao processo de tradução e adaptação transcultural (inglês para italiano). Um dos estudos encontrados apresentou de modo ambíguo os resultados de AFE em conjunto com ACP (ECR-M16; Tsilika et al., 2016). Como são análises diferentes, é comum que os resultados encontrados através de ACP ao serem comparados com uma AFE apresentem cargas maiores, assim como maior variância explicada e comunalidade. Isto ocorre, pois, as análises consideram variâncias diferentes, portanto ambas as análises não podem ser confundidas ou tomadas como idênticas (Brown, 2009; Damásio, 2012).

As demais versões e estudos da ECR apresentaram bons índices e métodos variados de análise, sendo este modelo de escala apresentado como uma forma de mensuração do apego amplamente utilizada. Entretanto, a estrutura com os fatores de apego ansioso e evitativo acabam limitando a exploração de outras nuances do apego, o mesmo ocorre com a URCS e sua estrutura de um fator para apego seguro, apesar de possuir bons índices de ajuste e consistência interna excelente. Neste sentido, a escala SAAM com três fatores que expressam apego seguro, ansioso e evitante parece surgir como um meio-termo entre ambas. Já as escalas ASQ, RSQ e ASPA-SF, abrangeram o maior número de estilos de apego, porém, a RSQ (Andersen et al., 2017) em específico demonstrou dificuldades de sustentação empírica para o modelo original com 4 fatores de Griffin e Bartholomew (1994), recorrendo à solução de 3 fatores alternativos.

Com relação aos índices de alfa é possível afirmar que as escalas encontradas apresentaram confiabilidade satisfatória, variando entre 0,70 a 0,90 indicando variação de confiabilidade das escalas de alta à forte. Vale ressaltar que a ASQ-24 apresentou o coeficiente de 0,58 para a subescala Dismissing, sendo este o valor mais baixo encontrado nos estudos. A escala unidimensional URCS (Vilar et al., 2017) apresentou o maior valor para confiabilidade, podendo ser considerado excelente $(0,94)$.

Realizando a integração dos resultados, as amostras variaram desde população geral e estudantes a pacientes com câncer e profissionais de saúde (RSQ; Andersen et al., 2017) ou pacientes em cuidados paliativos (ECR-M16, Tsilika et al., 2016). E mais importante, variaram em número fatores, desde um fator (URCS) como 
mencionado, até 5 fatores (ASPA-SF), sendo que algumas delas utilizaram nomenclaturas diversas para padrões de apegos similares, a exemplo: Dismissing nas escalas RSQ e ASQ-24 compreendida como uma forma de vinculação insegura, caracterizada por desdém em relação à figura de apego; Fearful nas escalas RSQ, ASQ-24, ASPA-SF compreendido como um padrão inseguro, caracterizado por medo e evitação; E Secure (ASQ-24, RSQ), Safe (ASPA-SF) e Security (SAAM), caracterizado por maior estabilidade emocional quanto a figura de apego (Griffin \& Bartholomew 1994).

Os modelos teóricos adotados também foram diversos e algumas escalas possuem como objetivo avaliar os tipos de apego tradicionais como podem ser visto nos trabalhos de Hazan e Shaver (1987) e Griffin e Bartholomew (1994), bem como estilos de apegos específicos ou contextualizados. Este é o caso do fator "Parentificação" da escala ASPA-SF (Michel \& Snow, 2019) que avalia a forma com que determinado sujeito percebe as relações de apego na infância, vale frisar que devido ao processo de resposta, tal fator pode sofrer viés de memória, assim como pode ocorrer em escalas que avaliam a relação em díades a exemplo, a escala ECRRS que avalia o apego com pai, mãe, companheiro íntimo e amigos (Ravitz, 2010). Em relação a PAM que avalia o apego em paciente com transtornos psicóticos, é importante considerar o nível de comprometimento do transtorno de modo que possa ser interessante utilizar outro método de avaliação (Ravitz, 2010; Souza et al., 2017).

É possível concluir que as escalas encontradas, são adequadas e demandam constante investigação de suas propriedades psicométricas, mas é relevante assinalar que apenas dois artigos utilizaram amostras brasileiras (URCS; Vilar et al., 2017; ECR-RS; Rocha et al., 2017). Deste modo, foi encontrada uma importante lacuna, nenhum dos instrumentos encontrados foi construído no Brasil e/ou avaliado com amostras de diferentes regiões, ou ainda realizados estudos de invariância, podendo este ser tema de trabalhos futuros. Apesar de ser uma temática bastante desenvolvida ao redor do mundo (Scrima, Rioux \& Lorito, 2014; Gumley, Taylor, Schwannauer \& MacBeth, 2013; Ravitz, Maunder, Hunter, Sthankiya \& Lancee, 2010), no Brasil, o interesse parece ainda estar em desenvolvimento, sendo necessários maiores esforços para proporcionar estudos com outras escalas, ou ainda a criação de um instrumento nacional.

Acerca das limitações do trabalho, é possível que as palavras-chave escolhidas tenham dificultado a composição da amostra, sendo muito relevante que futuras investigações possam recuperar um número maior de instrumentos. Outro fator importante a ser foi o direcionamento a instrumentos para adultos, é possível que existam outros instrumentos direcionados a outras faixas etárias.

\section{REFERÊNCIAS}

Ainsworth M. D, Blehar M. C, Waters E. \& Wall S. (1978). Patterns of attachment: a psychological study of the strange situation. Routledge: New York. 
*Andersen, C. M., Pedersen, A. F., Carlsen, A. H., Olesen, F., \& Vedsted, P. (2017). Data quality and factor analysis of the Danish version of the Relationship Scale Questionnaire. PLOS ONE, 12(5), e0176810. https://doi.org/10.1371/journal.pone.0176810

Barnum, E. L., \& Perrone, K. M. (2017). Attachment, Self-Esteem and Subjective Well-Being Among Survivors of Childhood Sexual Trauma. Journal of Mental Health Counseling, 39(1), 39-55. https://doi.org/10.17744/mehc.39.1.04

Bartholomew, K., \& Horowitz, L. M. (1991). Attachment styles among young adults: A test of a fourcategory model. Journal of Personality and Social Psychology, 61(2), 226-244. https://doi.org/10.1037//0022-3514.61.2.226

Berry, K., Wearden, A., Barrowclough, C., \& Liversidge, T. (2006). Attachment styles, interpersonal relationships and psychotic phenomena in a non-clinical student sample. Personality and Individual Differences, 41(4), 707-718. https://doi.org/10.1016/j.paid.2006.03.009

Blanton, H., M, L. J. (2018). Measurement in Social Psychology. Routledge.

Baptista, M. N., Tartaro, G. K., \& Peixoto, E. M. (2021) Teoria do Apego: conceituação, pesquisas e avaliação na infância e adolescência. In: M. Mansur-Alves; M. Muniz; D. S. Zanini; M. N. Baptista. (Org.). Avaliação Psicológica na Infância e Adolescência (pp.55-68). Rio de Janeiro, RJ: Vozes

Bowlby, J. (1969). Attachment and loss, Vol. 1: Attachment. New York: Basic Books.

Bowlby, J. (1973). Attachment and loss, Vol. 2: Separation. New York: Basic Books.

Bowlby, J. (1980). Attachment and loss, Vol. 3: Loss, sadness and depression. New York: Basic Books.

Brennan, K. A., Clark, C. L., \& Shaver, P. R. (1998). Self-report measurement of adult attachment: An integrative overview. In J. A. Simpson \& W. S. Rholes (Eds.), Attachment theory and close relationships (pp. 46-76). New York, NY, US: Guilford Press.

Brown, J. D. (2009). Statistics Corner Questions and answers about language testing statistics: Principal components analysis and exploratory factor analysis- Definitions, differences, and choices. Shiken: JALT Testing \& Evaluation SIG Newsletter, 13(2) 19-23. Recuperado de https://hosted.jalt.org/test/PDF/Brown30.pdf

*Busonera, A., Martini, P. S., Zavattini, G. C., \& Santona, A. (2014). Psychometric Properties of an Italian Version of the Experiences in Close Relationships-Revised (ECR-R) Scale. Psychological Reports, 114(3), 785-801. https://doi.org/10.2466/03.21.PR0.114k23w9

Cassidy, J., Jones, J. D., \& Shaver, P. R. (2013). Contributions of Attachment Theory and Research: A Framework for Future Research, Translation, and Policy. Development and psychopathology, 25(402), 1415-1434. https://doi.org/10.1017/S0954579413000692

Conselho Federal de Psicologia (2018), Resolução Nº09, de 25 de abril de 2018, Estabelece diretrizes para a realização de Avaliação Psicológica no exercício profissional da psicóloga e do psicólogo, regulamenta o Sistema de Avaliação de Testes Psicológicos - SATEPSI e revoga as Resoluções n 002/2003, n 006/2004 e n 005/2012 e Notas Técnicas n 01/2017 e 02/2017, Brasília, DF: Conselho Federal de Psicologia, Recuperado de: https://satepsi.cfp.org.br/docs/Resolu\%C3\%A7\%C3\%A3o-CFP-n\%C2\%BA-09-2018-comanexo.pdf

Damásio, B. F. (2012). Uso da análise fatorial exploratória em psicologia. Avaliação Psicológica, 11(2), 213-228. Recuperado

de http://pepsic.bvsalud.org/scielo.php?script=sci_abstract\&pid=S1677-04712012000200007.

Dibble, J. L., Levine, T. R., \& Park, H. S. (2012). The Unidimensional Relationship Closeness Scale (URCS) Reliability and validity evidence for a new measure of relationship closeness. Psychological Assessment, 24(3), 565-572. https://doi.org/10.1037/a0026265

Feeney, J. A., Noller, P., \& Hanrahan, M. (1994). Assessing adult attachment. In M. B. Sperling \& W. H. Berman (Eds.), Attachment in adults: Clinical and developmental perspectives (p. 128-152). New York: Guilford Press.

*Firoozabadi, A., Abedi, Z., Aliyari, R., Zolfaghari, B., \& Ghanizadeh, A. (2014). Psychometric Characteristics of the Persian (Farsi) Version of Attachment Style Questionnaire. Iranian Journal of Medical Sciences, 39(6), 506-514.

Fraley, R. C., Heffernan, M. E., Vicary, A. M., \& Brumbaugh, C. C. (2011). The experiences in close relationships-Relationship Structures Questionnaire: A method for assessing attachment orientations across relationships. Psychological Assessment, 23(3), 615-625. https://doi.org/10.1037/a0022898 
Fraley, R. C., Waller, N. G., \& Brennan, K. A. (2000). The Experiences in Close Relationships-Revised (ECR-R) Questionnaire. Psychological Assessment, 11(2), 705-725 https://doi.org/10.1037/0022-3514.78.2.350

Gillath, O., Hart, J., Noftle, E. E., \& Stockdale, G. D. (2009). Development and validation of a state adult attachment measure (SAAM). Journal of Research in Personality, 43(3), 362-373. https://psycnet.apa.org/doi/10.1016/j.jrp.2008.12.009

Greenwald, A. G., Nosek, B. A., \& Sriram, N. (2006). Consequential validity of the Implicit Association Test: Comment on Blanton and Jaccard (2006). American Psychologist, 61(1), 56-61. https://doi.org/10.1037/0003-066x.61.1.56

Griffin, D. W., \& Bartholomew, K. (1994). Models of the self and other: Fundamental dimensions underlying measures of adult attachment. Journal of personality and social psychology, 67(3), 430. https://psycnet.apa.org/doi/10.1037/0022-3514.67.3.430

Gumley, A. I., Taylor, H. E. F., Schwannauer, M., \& MacBeth, A. (2014). A systematic review of attachment and psychosis: Measurement, construct validity and outcomes. Acta Psychiatrica Scandinavica, 129(4), 257-274. https://doi.org/10.1111/acps.12172

Hox, J. J., \& Bechger, T. M. (1998). An introduction to structural equation modeling. Family Science Review, 11 (20), 354-373

Hu, L., \& Bentler, P. M. (1999). Cutoff criteria for fit indexes in covariance structure analysis: Conventional criteria versus new alternatives. Structural Equation Modeling: A Multidisciplinary Journal, 6(1), 1-55. https://doi.org/10.1080/10705519909540118

*Michael, T., \& Snow, M. (2019). The Adult Scale of Parental Attachment-Short Form: Psychometric Properties, Factor Analyses, and Validation. International Journal for the Advancement of Counselling, 41(4), 509-529. https://doi.org/10.1007/s10447-019-09375-9

Moshkani, M. \& Afrooz, G. (2018). Compare Big Five personality traits, attachment and self-perception between delinquent and normal adolescents. MEJDS, 20(19), 9:53. Recuperado de https://jdisabilstud.org/article-1-996-en.html

Muthén, L. K., \& Muthén, B. O. (2012). Mplus: Statistical analysis with latent variables; user's guide, [version 7]. Seventh Edition. Los Angeles, CA: Muthén \& Muthén

*Olbert, C. M., Penn, D. L., Reise, S. P., Horan, W. P., Kern, R. S., Lee, J., \& Green, M. F. (2016). Assessment of attachment in psychosis: A psychometric cause for concern. Psychiatry Research, 246(5), 77-83. https://doi.org/10.1016/j.psychres.2016.09.020

Özyurt, G., Öztürk, Y., Onat, M., Mutlu, C., \& Akay, A. (2018). Attachment, emotion regulation and anger expression in adolescent depression: Did comorbid anxiety disorder not have a role? Current Psychology, 246(5), 77-83. https:// https://doi.org/10.1007/s12144-018-9985-5

Ravitz, P., Maunder, R., Hunter, J., Sthankiya, B., \& Lancee, W. (2010). Adult attachment measures: A 25-year review. Journal of Psychosomatic Research, 69(4), 419-432. https://doi.org/10.1016/j.jpsychores.2009.08.006

*Rocha, G. M. A., Peixoto, E. M., Nakano, T. de C., Motta, I. F. da, \& Wiethaeuper, D. (2017). The Experiences in Close Relationships - Relationship Structures Questionnaire (ECR-RS): Validity evidence and reliability. Psico-USF, 22(1), 121-132. https://doi.org/10.1590/141382712017220111

Scrima, F., Rioux, L., \& Lorito, L. (2014). Three-Factor Structure of Adult Attachment in the Workplace: Comparison of British, French, and Italian Samples. Psychological Reports, 115(2), 627-642. https://doi.org/10.2466/49.pr0.115c25z2

Hazan, C., \& Shaver, P. (1987). Romantic love conceptualized as an attachment process. Journal of $\begin{array}{lll}\text { Personality and Social 5sychology, 52(3), } & \text { 524. }\end{array}$ https://psycnet.apa.org/doi/10.1037/0022-3514.52.3.511

Snow, M. S., Sullivan, K., Martin, E. E., \& Helm, H. (2005). The adult scale of parental attachment: Psychometric properties, factor analysis, and multidimensional scaling. Unpublished Manuscript, Department of Leadership and Counselor Education.

Souza, A. C. de, Alexandre, N. M. C., Guirardello, E. de B., Souza, A. C. de, Alexandre, N. M. C., \& Guirardello, E. de B. (2017). Propriedades psicométricas na avaliação de instrumentos: avaliação da confiabilidade e da validade. Epidemiologia e Serviços de Saúde, 26(3), 649-659. https://doi.org/10.5123/s1679-49742017000300022

Taber, K. S. (2018). The Use of Cronbach's A When Developing and Reporting Research Instruments in Science Education. Research in Science Education, 48(6), 1273-1296. https://doi.org/10.1007/s11165-016-9602-2 
*Tasca, G. A., Brugnera, A., Baldwin, D., Carlucci, S., Compare, A., Balfour, L., Proulx, G., Gick, M., \& Lafontaine, M.-F. (2018). Reliability and validity of the Experiences in Close Relationships Scale-12: Attachment dimensions in a clinical sample with eating disorders. International Journal of Eating Disorders, 51(1), 18-27. https://doi.org/10.1002/eat.22807

*Trentini, C., Foschi, R., Lauriola, M., \& Tambelli, R. (2015). The State Adult Attachment Measure (SAAM): A construct and incremental validity study. Personality and Individual Differences, 85(1), 251-257. https://doi.org/10.1016/j.paid.2015.05.016

*Tsilika, E., Parpa, E., Galanopoulou, N., Gennimata, V., Mosa, E., Galanos, A., \& Mystakidou, K. (2016) Attachment orientations of Greek cancer patients in palliative care. A validation study of the Experiences in Close Relationships scale (ECR-M16). JBUON 21(4) 1005-1012. Recuperado de https://jbuon.com/archive/21-4-1005.pdf

Vanhecke T. E. (2008). Zotero. Journal of the Medical Library Association: JMLA, 96(3), 275-276. https://dx.doi.org/10.3163/1536-5050.96.3.022

Varghese, M. E., \& Pistole, M. C. (2017). College Student Cyberbullying: Self-Esteem, Depression, Loneliness, and Attachment. Journal of College Counseling, 20(1), 7-21. https://doi.org/10.1002/jocc. 12055

*Vilar, R., Araujo, R. D. C., Coelho, G. L., Grangeiro, A. S., \& Gouveia, V. V. (2017). Psychometric Properties of the Unidimensional Relationship Closeness Scale (URCS) for a Brazilian Sample. The Spanish Journal of Psychology, 20(1), 1-24. https://doi.org/10.1017/sjp.2017.12

Woolley, M. E., Bowen, G. L., \& Bowen, N. K. (2004). Cognitive Pretesting and the Developmental Validity of Child Self-Report Instruments: Theory and Applications. Research on Social Work Practice, 14(3), 191-200. https://doi.org/10.1177/1049731503257882

Yip, J., Ehrhardt, K., Black, H., \& Walker, D. O. (2018). Attachment theory at work: A review and directions for future research. Journal of Organizational Behavior, 39(2), 185-198. https://doi.org/10.1002/job.2204

\section{CONFLITOS DE INTERESSES}

Os autores declaram não existir conflitos de interesses.

\section{SOBRE OS AUTORES}

Gustavo Kastien Tartaro é psicólogo, mestre e doutorando do Programa de Pós-Graduação stricto sensu em Psicologia da Universidade São Francisco.

E-mail: kastien.gustavo@gmail.com.

(2) https://orcid.org/0000-0002-2659-1430

Makilim Nunes Baptista é psicólogo, doutor em Psiquiatria e Psicologia Médica pela Universidade Federal de São Paulo. Atualmente é professor do Programa de Pós-Graduação stricto sensu em Psicologia da Universidade São Francisco e Bolsista Produtividade pelo Conselho Nacional de Desenvolvimento Científico e Tecnológico (CNPq).

E-mail: makilim01@gmail.com.

(2) https://orcid.org/0000-0001-6519-254X 